\title{
Rotavirus-Induced Neonatal Seizures with Cerebral White Matter Abnormalities on Magnetic Resonance Imaging: A Case Report
}

Ji Na Park, M.D., Hyun A Park, M.D., Yun Hye Shin, M.D., and Jong Hee Hwang, M.D.

Department of Pediatrics, Inje University College of Medicine, Ilsan Paik Hospital, Goyang, Korea

\section{ABSTRACT}

Rotavirus is a major cause of acute gastroenteritis in infancy and early childhood. Febrile seizures can occur in some infants or children exhibiting rotavirus gastroenteritis even without severe electrolyte imbalance, hypoglycemia or abnormal cerebrospinal fluid (CSF) finding. Some reports have described diffuse cerebral white matter lesions on diffusion-weighted magnetic resonance imaging (DW-MRI) in neonates with rotavirus-associated encephalopathy/encephalitis. In this case study, a 6-day-old male neonate was transferred to the neonatal intensive care unit after having a fever lasting 24 hours. On hospital day two, the seventh day after birth, the patient had his first seizure. The pregnancy and delivery were uneventful. The lab findings, including a CSF exam, were normal, but a stool antigen test for rotavirus was positive. The electroencephalography (EEG) examination result was normal. DWMRI of the brain showed bilateral symmetric diffusion restriction in the genu and splenium of the corpus callosum as well as in the periventricular white matter of the lateral ventricles. Multiple scattered high-signal-intensit foci on T1-weighted image/ fluid-attenuated inversion recovery (FLAIR) in the periventricular white matter were also seen bilaterally. He is now 17 months old, and there were no further seizures. He did not show any neurodevelopmental delay. This case reports that the patient with rotavirus-induced neonatal seizures with cerebral white matter abnormalities on magnetic resonance imaging (MRI) showed a normal neurodevelopmental outcome on the follow-up.

Key words: Rotavirus, Seizure, Febrile, Diffusion magnetic resonance imaging, White matter

\section{INTRODUCTION}

Rotavirus is a major cause of acute gastroenteritis in infancy and early childhood ${ }^{1)}$. The disease is usually self-limiting; however, febrile seizure can occur in some infants or children with rotavirus gastroenteritis even without severe electrolyte imbalance, hypoglycemia or
Received: 22 September 2016

Revised: 22 November 2016

Accepted: 30 November 2016

Correspondence to: Jong Hee

Hwang

Department of Pediatrics, Inje

University College of Medicine, Ilsan Paik Hospital, 170 Juhwa-ro, Ilsanseo-gu, Goyang 10380, Korea

Tel: +82-31-910-7114

Fax: +82-31-910-7108

E-mail: jhhwang@paik.ac.kr

Copyright(c)

By Korean Society of Neonatology.

All right reserved.

This is an Open-Access article distributed under the terms of the Creative Commons Attribution Non-Commercial License (http://creativecommons.org/licenses/ by-nc/4.0), which permits unrestricted non-commercial use, distribution, and reproduction in any medium, provided the original work is properly cited. 
abnormal cerebrospinal fluid (CSF) finding ${ }^{2}$.

Several reports have shown that rotavirus can also cause diverse central nervous system(CNS) complications such as benign convulsions with gastroenteritis, encephalopathy/encephalitis and cerebellitis ${ }^{1-6)}$. Further, some reports have described diffuse cerebral white matter lesions on diffusion-weighted magnetic resonance imaging (DW-MRI) in neonates with rotavirus-associated febrile seizures, encephalopathy and encephalitis ${ }^{2,3,5,6)}$. Here, we report on a neonatal patient with rotavirus-induced febrile seizure, exhibiting white matter lesions on DW-MRI.

\section{CASE REPORT}

A 6-day-year old male neonate was transferred to the neonatal intensive care unit with a fever lasting 24 hours. Upon arrival at the neonatal intensive care unit (ICU), the patient's body temperature was between $37.7-38.2^{\circ} \mathrm{C}$. The patient exhibited no symptoms other than a mild fever. He was born by a normal delivery, was the first child of the family, and weighed $3.55 \mathrm{~kg}$ at birth. The gestational age was 40 and $1 / 7$ weeks. The Apgar scores were 9 and 10, at 1 and 5 minutes, respectively. No significant events occurred during the delivery. The 35-year old mother had been taking Synthroid to treat hypothyroidism diagnosed during pregnancy. No maternal history of smoking, alcohol or teratogenic exposure was found. A prenatal ultrasonography revealed no fetal abnormalities. At birth, the patient was clinically stable. Symptoms such as fever, vomiting, diarrhea, apnea and others did not appear for 5 days from the day of birth.

The patient's initial vital sign showed a blood pressure of $66 / 41$
$\mathrm{mmHg}$, a pulse of 160 beats $/ \mathrm{min}$, a body temperature of $38.2^{\circ} \mathrm{C}$ and respiratory rate of 42 breaths/min. His body weight was $3.54 \mathrm{~kg}$. A physical exam revealed a cephalhematoma of $6 \times 7 \mathrm{~cm}$ on the right parietal lobe. Otherwise, physical and neurological exams did not show any abnormal signs. The muscle tone and reflexes were also normal. On hospital day two, the seventh day after birth, the patient developed his first seizure. The seizures lasted about 15 seconds, beginning with unilateral clonic movements of the upper right arm and spreading to lower limbs. They were difficult to categorize but were generalized seizures. At that time, there was no fever. The seizure was treated with phenobarbital $1.5 \mathrm{mg} / \mathrm{kg}$ per day, and there were no further clinical seizures. Laboratory tests revealed a white blood cell count of $7,420 / \mathrm{mm}^{3}$, a hemoglobin level of $13.9 \mathrm{~g} / \mathrm{dL}$, and a platelet count of $163,000 / \mathrm{mm}^{3}$. Test showed a C-reactive protein level of $0.2 \mathrm{mg} / \mathrm{L}$ and electrolyte levels of $138 \mathrm{mmol} / \mathrm{L}\left(\mathrm{Na}^{+}\right)$, $4.9 \mathrm{mmol} / \mathrm{L}\left(\mathrm{K}^{+}\right)$and $109 \mathrm{mmol} / \mathrm{L}\left(\mathrm{Cl}^{-}\right)$. Glucose was $77 \mathrm{mg} / \mathrm{dL}$, protein was $5.23 \mathrm{mg} / \mathrm{dL}$, albumin was $3.29 \mathrm{mg} / \mathrm{dL}$, aspartate aminotransferase was $32 \mathrm{U} / \mathrm{L}$, alanine aminotransferase was 12 $\mathrm{U} / \mathrm{L}$, blood urea nitrogen was $9 \mathrm{mg} / \mathrm{dL}$, serum Ca $8.4 \mathrm{mmol} / \mathrm{L}$, and phosphate was $5.9 \mathrm{mg} / \mathrm{dL}$. A stool antigen test was positive for rotavirus. The newborn screening test (NST) result was within the normal range. The CSF studies revealed 5 leukocytes $/ \mathrm{mm}^{3}$ (granulocytes $40 \%$, lymphocytes $60 \%$ ) with $62 \mathrm{mg} / \mathrm{dL}$ protein and $47 \mathrm{mg} / \mathrm{dL}$, glucose. CSF bacterial cultures were negative. The electroencephalography (EEG) finding was normal (Figure 1). And brain sonography showed no abnormal lesions. DW-MRI of the brain on hospital day thirteen showed bilateral symmetric diffusion restriction in genu and splenium of corpus callosum as well as in the periventricular white matter of the lateral ventri-

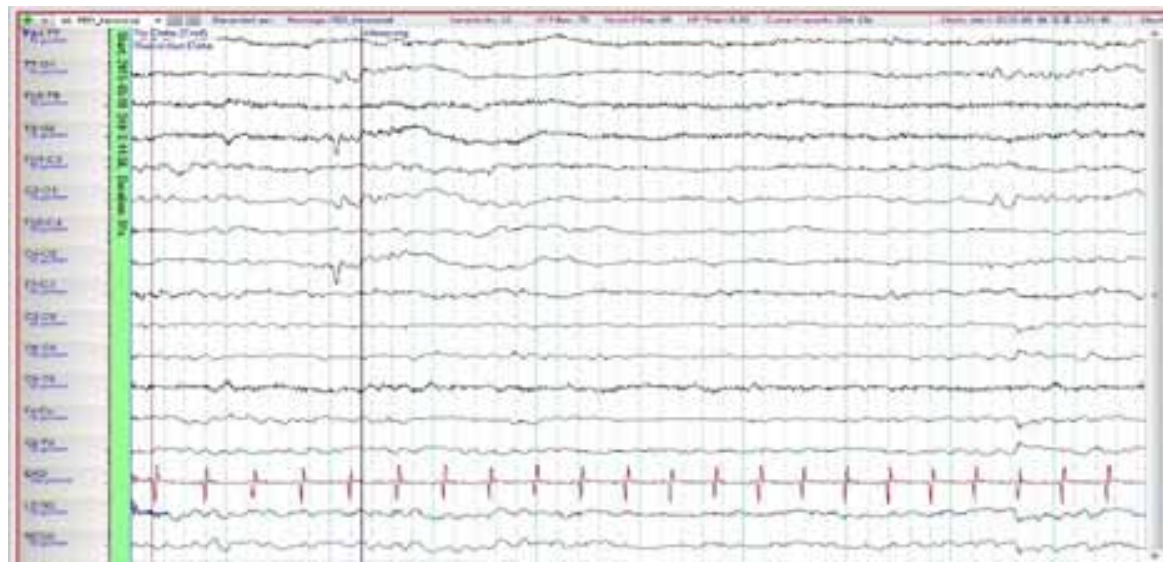

Figure 1. The EEG showed normal findings on the eighth day after birth. Abbreviation: EEG, electroencephalography. 


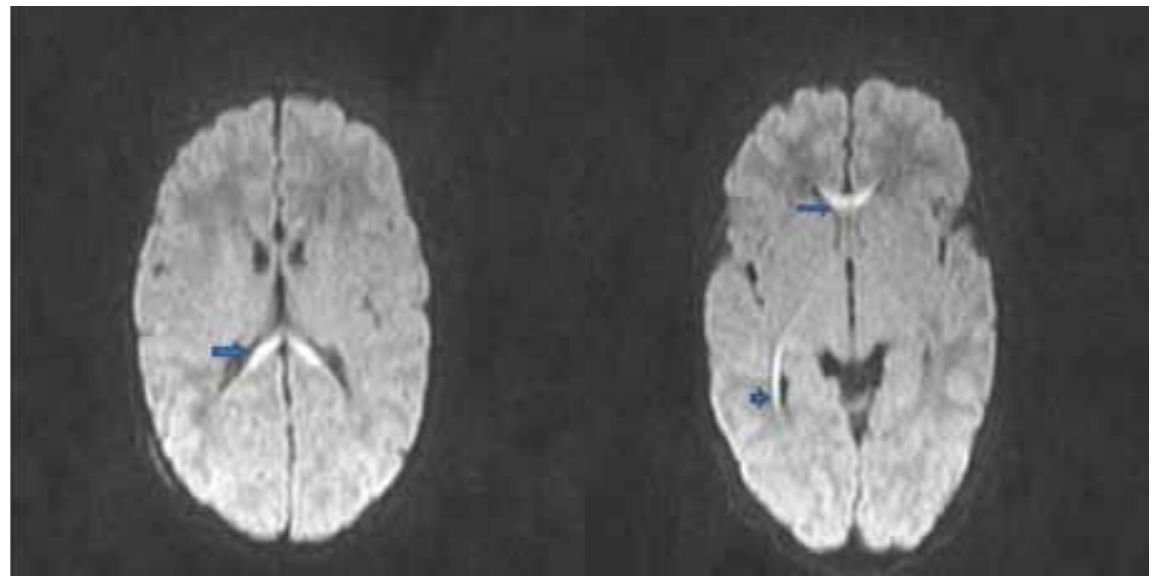

Figure 2. Diffusion-weighted magnetic resonance imaging on the sixteenth day after birth. This showed bilateral symmetric diffusion restriction in genu/splenium of corpus callosum and both periventricular white matter of lateral ventricles (arrows).

cles. Multiple scattered high-signal-intensity foci on T1-weighted images/fluid-attenuated inversion recovery (FLAIR) in the periventricular white matter were also seen bilaterally (Figure 2).

At 17 days of age he was discharged in a good general condition. But Phenobarbital medication per oral was continued for 4 months

Follow-up brain MRIs were performed at the age of 8 months and 16 months. At 8 months, brain MRI showed interval regression of multifocal scattered high S1 foci on T1-weighted images/ FLAIR in both periventricular white matter. And a repeat brain MRI was obtained recently at the age of 16 months, showed residual focal high S1 on T2-weighted images/FLAIR and low S1 on T1-weighted images in both frontal and parietotemporal periventricular white matter. He is now 17 months old and does not show a recurrence of seizures. He does not show any motor and speech delay. His neurodevelopment is evaluated regularly at rehabilitation medicine clinic.

\section{DISCUSSION}

Rotavirus is a common cause of seizures, either febrile or afebrile, in young children ${ }^{2}$. Rotavirus can cause seizures not only in infants with underlying neurologic or systemic conditions, but also in otherwise healthy infants ${ }^{7}$.

Some reports also suggested that rotaviral infection should be considered in neonates presenting seizures accompanied by diffuse cerebral white matter lesions shown on DW-MRI ${ }^{3,5,6}$. According to Lee et al., full-term neonates presenting with sei- zures exhibited with the following characteristics on brain DWMRI: diffuse diffusion-restricted lesions in bilateral cerebral white matter, including the corpus callosum, and no cerebral cortical lesions $^{2)}$. No patients developed a fever or skin rash, and $30.8 \%$ of patients had diarrhea. They reported that all patients with rotavirus-induced neonatal seizures with cerebral white matter injury on MRI showed abnormal EEGs. Some patients showed cerebral atrophy or cerebromalacia in white matter on follow-up MRI and a motor and speech delay. Otherwise, this case showed the neonate with rotavirus-induced seizures had a normal EEG. Our patient showed regression on follow-up MRI and a normal neurodevelopment.

Another report described that all infants with rotavirusinduced seizures had normal CSF cell counts and normal protein and glucose levels. Seventy five percent of these infants, especially preterm infants ${ }^{8)}$, developed late-onset cystic periventricular leukomalacia and a neurodevelopmental delay. In some reports, rotavirus nucleic acid and rotavirus-specific immunoglobulin $\mathrm{G}$ have been detected in the CSF of children with afebrile seizures, confirming that rotavirus can invade the central nervous system $^{1,9)}$. However, rotavirus antigen and/or polymerase chain reaction (PCR) were not always positive in encephalitis/encephalopathy associated rotavirus gastroenteritis ${ }^{10)}$. Therefore more studies is needed for the pathophysiology of CNS infection associated rotavirus infection.

There was a limitation to the present study. Rotavirus antigen examination was not performed in CSF. But according to Oh et al., mostly rotavirus antigen was not detected in CSF and serum samples ${ }^{5)}$. Therefore, we suggest that infants with clinically 
rotavirus-induced seizures should be evaluated by periodic MRI and long-term follow-up, especially preterm infants.

\section{REFERENCES}

1) Hung JJ ,Wen HY, Yen MH, Chen HW, Yan DC, Lin KL, et al. Rotavirus gastroenteritis associated with afebrile convulsion in children: clinical analysis of 40 cases. Chang Gung Med J 2003; 26:654-9.

2) Lee KY, Oh KW, Weon YC, Choi SH. Neonatal seizures accompanied by diffuse cerebral white matter lesions on diffusionweighted imaging are associated with rotavirus infection. Eur J Paediatr Neurol 2014;18:624-31.

3) Kubota T, Suzuki T, Kitase Y, Kidokoro H, Miyajima Y, Ogawa A, et al. Chronological diffusion-weighted imaging changes and mutism in the course of rotavirus-associated acute cerebellitis/ cerebellopathy concurrent with encephalitis/ encephalopathy. Brain Dev 2011;33:21-7.

4) Lynch M, Lee B, Azimi P, Gentsch J, Glaser C, Gilliam S, et al. Rotavirus and central nervous system symptoms: cause or contaminant? Case reports and review. Clin Infect Dis 2001;33: 932-8.

5) Oh KW, Moon CH, Lee KY. Association of rotavirus with seizures accompanied by cerebral white matter injury in neonates. J Child Neuro 2015;30:1433-9.

6) Yeom JS, Kim YS, Seo JH, Park JS, Park ES, Lim JY, et al. Distinctive pattern of white matter injury in neonates with rotavirus infection. Neurology 2015;84:21-7.

7) Lloyd MB, Lloyd JC, Gesteland PH, Bale JF Jr. Rotavirus gastroenteritis and seizures in young children. Pediatr Neurol 2010;42:404-8.

8) Verboon-Maciolek MA, Truttmann AC, Groenendaal F, Skranes J, Døllner H, Hunt RW, et al. Development of cystic periventricular Leukomalacia in newborn infants after rotavirus Infection. J Pediatr 2012;160:165-8.el.

9) Hongou K, Konishi T, Yagi S, Araki K, Miyawaki T. Rotavirus encephalitis mimicking afebrile benign convulsions in infants. Pediatr Neurol 1998;18:354-7.

10) Nakagomi $T$, Nakagomi $O$. Rotavirus antigenemia in children with encephalopathy accompanied by rotavirus gastroenteritis. Arch Virol 2005;150:1927-31. 